\title{
Tissue oxygenation as a target for goal-directed therapy in high-risk surgery: a pilot study
}

\author{
Paul A van Beest ${ }^{*}$, Jaap Jan Vos, Marieke Poterman, Alain F Kalmar and Thomas WL Scheeren
}

\begin{abstract}
Background: Tissue hypoperfusion occurs frequently during surgery and may contribute to postoperative organ dysfunction. There is a need for perioperative treatment protocols aiming at improving tissue oxygenation $\left(\mathrm{StO}_{2}\right)$. We hypothesised that intra-operative optimisation of $\mathrm{StO}_{2}$ improves tissue perfusion and thus reduces postoperative complications. Furthermore, we evaluated the feasibility of the optimisation algorithm used.

Methods: We randomized 50 high-risk patients, all >65 years with ASA physical status III, who underwent major abdominal surgery under standardized balanced general anesthesia combined with epidural analgesia. Throughout surgery $\mathrm{StO}_{2}$ was monitored at the thenar eminence using near-infrared spectroscopy. All patients were treated according to a standard care algorithm. In addition, patients in the intervention group were treated with dobutamine if necessary to keep or raise $\mathrm{StO}_{2} \geq 80 \%$. Data were recorded continuously and complications were recorded during hospital stay with a maximum of 28 days.

Results: The number of complications was not significantly different between groups (11 vs 20; $p=0.23$ ). Eleven patients in the intervention group had no complication, versus 7 in the control group. There was no significant difference between groups in length of stay in ICU or in hospital. Only ten patients in the intervention group received dobutamine. Administration of dobutamine resulted in a moderate $6[-3$ to 10$] \%$ increase of $\mathrm{StO}_{2}$. The overall protocol adherence was $94 \%$.
\end{abstract}

Conclusions: No statistically significant difference in outcome was realized through intraoperative optimization of $\mathrm{StO}_{2}$ values in this pilot study. The protocol used may be considered feasible for clinical practice. Further research is obligatory to define both the optimal $\mathrm{StO}_{2}$ threshold and intervention to treat tissue hypoperfusion.

Trial registration: ClinicalTrials.gov identifier: NCT01342900. Registered 21 April 2011.

Keywords: Tissue oxygenation, Near-infrared spectroscopy, Postoperative complications, High-risk surgery, Goal-directed therapy

\section{Background}

Postoperative complications determine long-term survival after high-risk surgery. Although high-risk surgical patients account for a minority of surgical procedures they represent a vast majority of post-operative mortality rates [1]. Therefore, a decrease in post-operative complications is important and can be achieved by using goal-directed fluid optimization [2]. In order to limit post-operative complications, adequate monitoring and optimisation of physiological variables during surgery are necessary.

\footnotetext{
*Correspondence: p.van.beest@umcg.nl

Department of Anesthesiology, University Medical Center Groningen,

University of Groningen, PO Box 30001, 9700 RB Groningen, the Netherlands
}

Hemodynamic variables such as heart rate, arterial pressure and central venous pressure are commonly used but have been proven to be poor predictors of outcome. In contrast, the use of flow-related variables such as cardiac index $(\mathrm{CI})$, oxygen delivery $\left(\mathrm{DO}_{2}\right)$ and central venous oxygen saturation $\left(\mathrm{ScvO}_{2}\right)$ have been associated with improved outcome after high-risk surgery [3]. Improvement of these hemodynamic and oxygen derived variables may affect tissue oxygenation and as a result preserve organ function. While these variables reflect global changes in either the upstream or the downstream part of the circulation and not regional changes as tissue oxygenation, they may be of vital importance since undetected tissue hypoxia may contribute to postoperative organ dysfunction. 
Remarkably, even though tissue hypoperfusion occurs frequently during high-risk surgery or in high-risk patients undergoing surgery $[4,5]$ there is a lack of protocols for the perioperative management of high-risk surgical patients [6].

The purpose of the present interventional pilot study is primarily to evaluate the effect of the use of a protocol based on optimisation of tissue oxygenation $\left(\mathrm{StO}_{2}\right)$ on the incidence of perioperative complications. As a secondary objective, we assessed the feasibility of such optimisation protocol in clinical practice.

\section{Methods}

The study complied with the Declaration of Helsinki. The protocol was approved by the Local Ethics Committee (Medisch Ethische Toetsingscommissie UMC Groningen; METc 2010/209) and the study was registered on ClinicalTrials.gov (NCT01342900). Written informed consent was obtained from all participants during the pre-anesthetic consultation.

\section{Patients}

Eligible patients were ASA III and IV patients of 65 years and older who were scheduled to undergo elective major surgery under general anesthesia combined with epidural analgesia.

Exclusion criteria were patients undergoing emergency surgery, refusal, neurosurgical patients, and patients with disseminated malignancy or receiving palliative treatment only. Patients undergoing extensive liver surgery requiring low central venous pressure (CVP) management and patients scheduled for a fast track protocol were excluded as well.

\section{Anesthetic technique}

The patients received a standardized balanced anesthesia. Each patient received acetaminophen $1000 \mathrm{mg}$ orally as premedication. Standard vital signs monitors were placed, including Bispectral monitoring [BIS, Aspect Medical Systems, Norwood, USA]. Thoracic epidural catheter was placed by standard procedure. Anesthesia was induced using target-controlled infusion (TCI) of sufentanil with an effect site concentration $\left(C_{\mathrm{e}} \mathrm{t}\right)$ of $15 \mathrm{ng} \mathrm{ml}^{-1}$ and TCI of propofol (2-4 $\left.\mu \mathrm{g} \mathrm{ml}^{-1} \mathrm{C}_{\mathrm{e}} \mathrm{t}\right)$ according to institutional practice, adjusted if necessary to keep BIS in the range of 40-60. After loss of verbal contact and confirmation of adequate mask ventilation, rocuronium $\left(0.6 \mathrm{mg} \mathrm{kg}^{-1}\right)$ was administered and the patient's trachea was intubated. Patients were mechanically ventilated (volume controlled mode) with a tidal volume of $7 \mathrm{ml} \mathrm{kg}^{-1}$ body weight, unless required otherwise, i.e. one lung ventilation during oesophagotectomy. Subsequently, TCI sufentanil was reduced to $0.10 \mathrm{ng} \mathrm{ml}^{-1} \mathrm{C}_{\mathrm{e}} \mathrm{t}$. A bolus of 6-8 ml $0.25 \%$ levobupivacaine and $20-30 \mu \mathrm{g}$ sufentanil was given epidurally before start of surgery, and at skin incision sufentanil was increased to $0.20 \mathrm{ng} \mathrm{ml}^{-1} \mathrm{C}_{\mathrm{e}}$. Epidural analgesia with $0.125 \%$ levobupivacaine $+1 \mu \mathrm{g} \mathrm{ml}^{-1}$ sufentanil was maintained at a rate of $6-8 \mathrm{ml} \mathrm{h}^{-1}$. A radial or brachial arteria was cannulated for continuous measurement of the arterial pressure and was connected to the FloTrac Vigileo system (Version 03.02; Edwards Lifesciences LLC, Irvine, CA, USA) for measuring cardiac output (CO). All patients received a central venous line (jugular vein) with continuous oximetry (PreSep catheter; Edwards Lifesciences LLC, Irvine, CA, USA) for measuring $\mathrm{ScvO}_{2}$.

\section{Study protocol}

$\mathrm{StO}_{2}$ was monitored non-invasively in all patients by near infrared spectroscopy using the InSpectra System [Model 650; Hutchinson Technology, Hutchinson, MN, USA]. The device consists of a monitor and a sensor, which is placed on the thenar eminescence of the patient. In the control group the anesthetist/investigator was blinded for data from the InSpectra monitor. All patients were treated according to the standard care algorithm (Figure 1). No dobutamine was to be administered in the control group. In addition, patients in the intervention group were treated according to the intervention algorithm aiming to keep or raise the $\mathrm{StO}_{2} \geq 80 \%$ (Figure 1). If the patient's heart rate exceeded 110 beats per minute or if new rhythm disturbances occurred during dobutamine infusion it was left to the anesthesist's discretion not to give or not to increase dobutamine.

\section{Randomisation}

After induction of anesthesia, but prior to the start of the surgery, patients were randomly assigned to the control group or the intervention group using sealed envelopes.

\section{Follow-up}

Upfront, all patients were scheduled to be postoperatively monitored either at an intensive care unit (ICU) or at a prolonged stay at the post-anesthesia care unit (PACU). All PACU and ICU teams were blinded for patients' allocation. Follow-up for postoperative complications continued during the total hospital stay with a maximum of 28 days.

\section{Data collection}

All standard data from the anesthesia monitor (MP70, Philips, Eindhoven, NL) was recorded continuously (sampling rate of $1 \mathrm{~Hz}$ ) utilizing data-logging software (RugLoop II, Demed Engineering, Temse, Belgium) on a medical grade Windows XP based personal computer. The $\mathrm{StO}_{2}$ data of the InSpectra ${ }^{\mathrm{Tm}}$ device and the hemodynamic variables were imported into Microsoft Excel $2010^{\circ}$ (Microsoft, Redmond, WA, USA) for synchronisation and analysis. After graphical representation, a visual inspection of the 


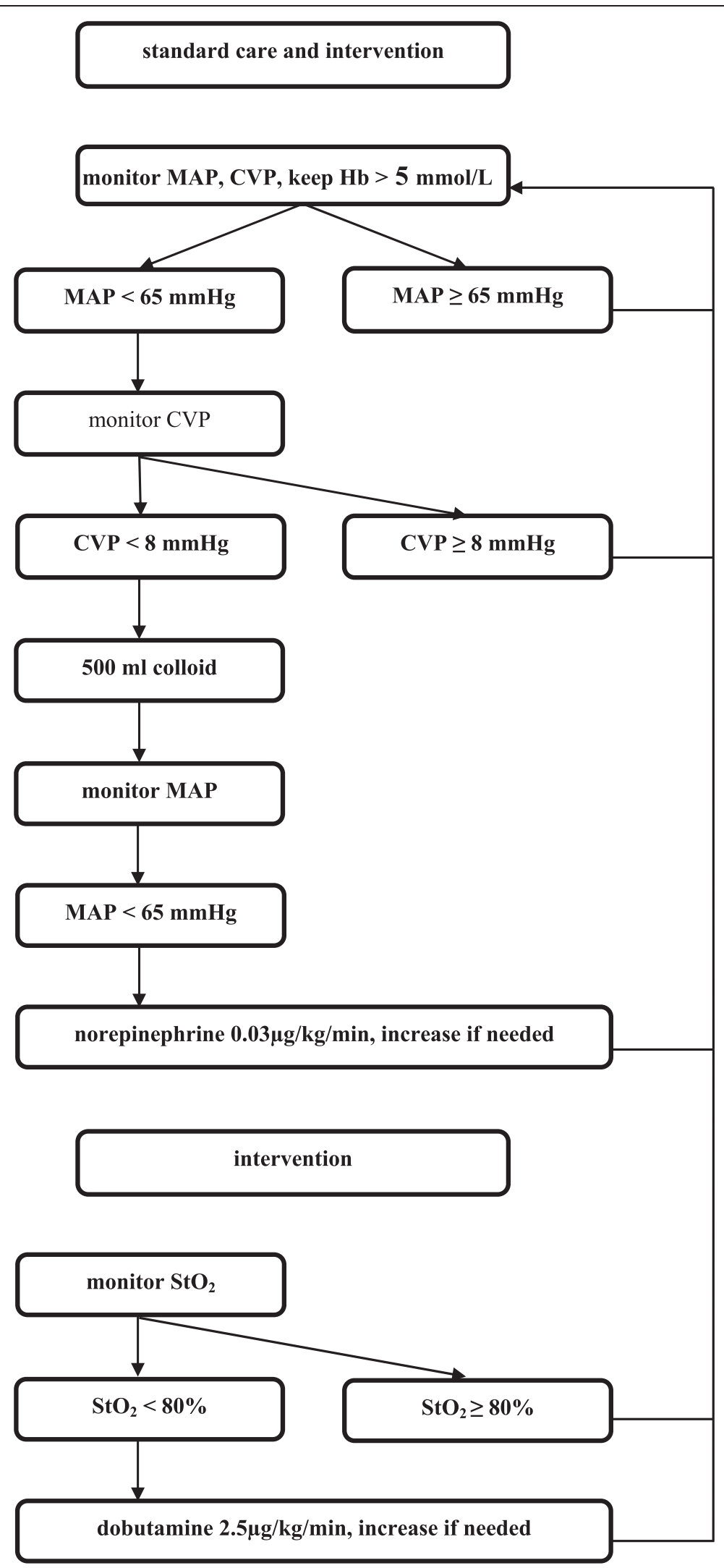

Figure 1 Standard care and intervention algorithm. MAP, mean arterial pressure; CVP, central venous pressure; $\mathrm{Hb}$, hemoglobin; $\mathrm{StO}_{2}$, tissue oxygenation. 
data plots was performed to delete obvious atypical values caused by artifacts. Next, a 30-second moving median with 15-second steps was calculated for all studied variables. The evolution of the absolute values was plotted from 10 minutes before the start of dobutamine administration until 45 minutes thereafter.

All commonly used variables were recorded, including ventilator settings. In addition, patient characteristics, surgical information, fluid administration, and use of inotropes were collected. To prevent a ruffled picture on length of stay numbers due to the logistic reality of the PACU or the ICU, 'fit for discharge' criteria were noted every 6 hours during PACU/ICU stay. These criteria were: spontaneous breathing, oxygen saturation $\left(\mathrm{SpO}_{2}\right)>92 \%$ with $<3$ litre $\mathrm{O}_{2} /$ minute, systolic arterial pressure $>100 \mathrm{~mm} \mathrm{Hg}$ without vasoactive or inotropic support, and temperature in range of $36-38.5^{\circ} \mathrm{C}$. The patients were considered fit for discharge when all criteria were fulfilled. Both the therapeutic intervention scoring system (TISS)-score and sequential organ failure assessment (SOFA)-score were noted for insight in resource utilization and the degree of organ failure.

\section{Statistical analysis}

A priori, the population was divided into two groups: control group vs. intervention group. In addition, a subgroup from the intervention group was analyzed, i.e. patients in whom dobutamine was actually used (Dobu ${ }^{+}$ subgroup). Two-tailed statistical tests were performed by the statistical package for the social sciences (IBM SPSS 20 for Windows, Chicago, IL, USA). GraphPad software (Prism 5.0, La Jolla, CA, USA) was used for graphics. All continuous data were tested for normal distribution with the D'Agostino-Pearson omnibus normality test before further statistical analysis. Differences between groups were assessed using Student's $t$-test or Wilcoxon-MannWhitney test where appropriate. For categorical data Chi-Square test or Fisher's exact test was applied. Statistical significance was assumed at $\mathrm{p}<0.05$. Non-parametric data are presented as median [quartiles]. G Power was used to calculate a sample size from differences in the number of post-operative complications between the two study groups [7].

\section{Results}

\section{Patient characteristics}

A total of fifty patients were included and randomized of whom ten patients had to be excluded from the study. The reasons for exclusion were protocol deviation $(n=2$; poor use of equipment), protocol violation $(n=1$; use of dobutamine in control group), meeting exclusion criteria peri-operatively $(n=7$; disseminated malignancy or unable to place epidural catheter). As a result, forty patients were included for analysis (Figure 2).

Table 1 displays patient characteristics and intraoperative characteristics. Comorbidities were similar in both groups. All patients were scored grade III ASA physical status classification. No significant differences between the two groups with respect to type of surgery and administered fluids during surgery were noticed.

\section{Tissue oxygenation}

Baseline $\mathrm{StO}_{2}$ was 85 [82-90]\% in all patients. In the control group $(\mathrm{n}=20)$ the median $\mathrm{StO}_{2}$ value was 83 [77-87]\%. In eleven of these cases $\mathrm{StO}_{2}$ values showed a decline below the threshold of $80 \%$. Median $\mathrm{StO}_{2}$ values were $<80 \%$ in seven patients in the control group.

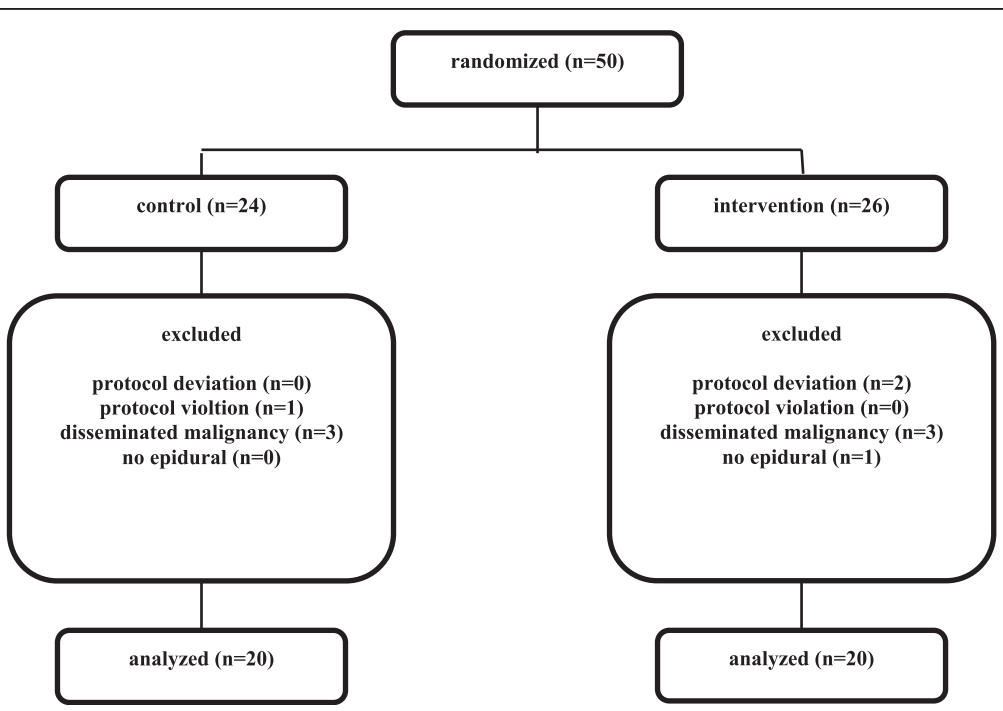

Figure 2 Flowdiagram of the study. 
Table 1 Patient baseline and intraoperative characteristics $(n=40)$

\begin{tabular}{|c|c|c|c|c|c|}
\hline Characteristics & Control $(n=20)$ & Intervention $(\mathrm{n}=20)$ & P-value* & $\operatorname{Dobu}^{+}(n=10)$ & P-value ${ }^{\S}$ \\
\hline Age (yr) & 75 [69-78] & 71 [69-78] & & $71[69-81]$ & \\
\hline Gender (M/F) & $16 / 4$ & $16 / 4$ & & $9 / 1$ & \\
\hline Body Mass Index $\left(\mathrm{m} / \mathrm{kg}^{2}\right)$ & 27 [22-31] & $25[23-27]$ & & 25 [23-28] & \\
\hline Smoking status (\%) & 60 & 35 & & 40 & \\
\hline \multicolumn{6}{|l|}{ Medical history (n) } \\
\hline COPD & 4 & 4 & & 1 & \\
\hline CAD & 5 & 9 & & 5 & \\
\hline $\mathrm{CHF}$ & 5 & 3 & & 2 & \\
\hline IDDM & 3 & 6 & & 3 & \\
\hline TIA & 2 & 2 & & 1 & \\
\hline Hemoglobine (g/dl) & $12.2[10.6-14.5]$ & $13.7[12.6-15.3]$ & 0.34 & 13.4 [10.6-15.6] & 0.25 \\
\hline Type of surgery (n) & & & 0.18 & & 0.13 \\
\hline AAA & 9 & 5 & & 2 & \\
\hline Oesophagus & 1 & 2 & & 2 & \\
\hline Stomach & 1 & 3 & & 1 & \\
\hline Pancreas & 1 & 5 & & 4 & \\
\hline Colon & 3 & 2 & & 0 & \\
\hline Other & 5 & 3 & & 1 & \\
\hline Duration surgery (min) & $277[221-334]$ & $262[145-387]$ & 0.63 & 349 [246-500] & 0.35 \\
\hline Blood loss (ml) & 800 [350-1575] & 375 [200-950] & 0.07 & 550 [288-1050] & 0.22 \\
\hline RBC transfusion (ml) & 0 [0-300] & $0[0-0]$ & 0.06 & 0 [0-150] & 0.32 \\
\hline Colloids (ml) & 1000 [563-1000] & 500 [0-1500] & 0.40 & 1000 [500-1625] & 0.83 \\
\hline Crystalloids (ml) & 3000 [2350-3875] & 2725 [2075-3500] & 0.80 & $3400[2500-4125]$ & 0.57 \\
\hline Norepinephrine (\%) & 50 & 75 & & 90 & \\
\hline Hemoglobine (g/dl) & $10.8[9.2-12.6]$ & 10.9 [9.9-12.3] & 0.73 & $11.4[9.3-12.4]$ & 0.74 \\
\hline $\mathrm{StO}_{2}$ - baseline (\%) & 86 [83-90] & 86 [81-90] & 0.68 & 81 [78-84] & 0.01 \\
\hline $\mathrm{StO}_{2}$ - average (\%) & 83 [77-87] & 85 [78-91] & 0.45 & 78 [74-82] & 0.07 \\
\hline $\mathrm{StO}_{2}-$ minimum (\%) & 71 [59-79] & 76 [70-82] & 0.22 & $70[57-72]$ & 0.40 \\
\hline $\mathrm{ScvO}_{2}$ - baseline (\%) & 80 [70-85] & $81[75-84]$ & 0.91 & 75 [74-83] & 0.53 \\
\hline $\mathrm{ScvO}_{2}$ - average (\%) & 77 [70-81] & 81 [77-83] & 0.09 & 78 [72-83] & 0.35 \\
\hline $\mathrm{ScvO}_{2}-$ minimum (\%) & $61[55-68]$ & 70 [59-72] & 0.13 & $65[57-72]$ & 0.38 \\
\hline
\end{tabular}

$\mathrm{Dobu}^{+}$, subgroup of intervention subjects that did receive dobutamine; COPD, chronic obstructive pulmonary disease; CAD, coronary artery disease; CHF, chronic

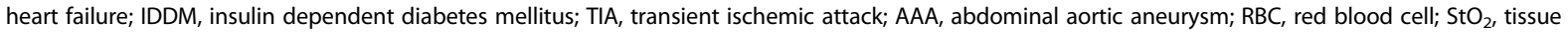
oxygenation; $\mathrm{ScvO}_{2}$, central venous oxygen saturation; baseline: start surgery; ${ }^{*}$ Control versus Intervention; ${ }^{\S}$ Control versus Dobu ${ }^{+}$.

No difference was found between the median intraoperative $\mathrm{StO}_{2}$ values of the control group and intervention group (83 [77-86.5]\% vs. 82.5 [78-90]\%; $\mathrm{p}=0.83)$. In half of the patients randomized for the intervention group $\mathrm{StO}_{2}$ values showed a drop below the predefined threshold of $80 \%$. Based on the study protocol dobutamine was administrered in those ten cases $\left(\mathrm{Dobu}^{+}\right.$subgroup; Table 1). Maximum median dobutamine dose was 4.9 [4.3-7.0] $\mathrm{\mu g} \mathrm{kg}^{-1} \mathrm{~min}^{-1}$. The predefined maximum dose of $12.5 \mu \mathrm{g} \mathrm{kg}^{-1} \mathrm{~min}^{-1}$ was never reached. In one case dobutamine dosage was limited $\left(10 \mu \mathrm{g} \mathrm{kg}^{-1} \mathrm{~min}^{-1}\right)$ since elevation in heart rate was considered too high $(>100$ beats per minute) by the attending anesthesist. Figure 3 shows the graphs of the continuously measured $\mathrm{StO}_{2}$ values in the $\mathrm{Dobu}^{+}$subgroup. Dobutamine induced a 6 [-3 to 10]\% increase in $\mathrm{StO}_{2}$, a 0.3 [0.0-0.6] litre $\mathrm{min}^{-1} \mathrm{~m}^{-2}$ increase in $\mathrm{CI}$, and a 4 [0 to 7$] \%$ increase in $\mathrm{ScvO}_{2}$ (Figure 3).

In the control group 9 patients (3/9 aorta surgery) received red blood cell (RBC) transfusions (range 300$3400 \mathrm{ml}$ ). Three patients from the intervention group (3/3 aorta surgery) received RBC transfusions (range 600-1800 ml). Two patients in the $\mathrm{Dobu}^{+}$subgroup received $600 \mathrm{ml} \mathrm{RBC}$ transfusions. There was no significant 


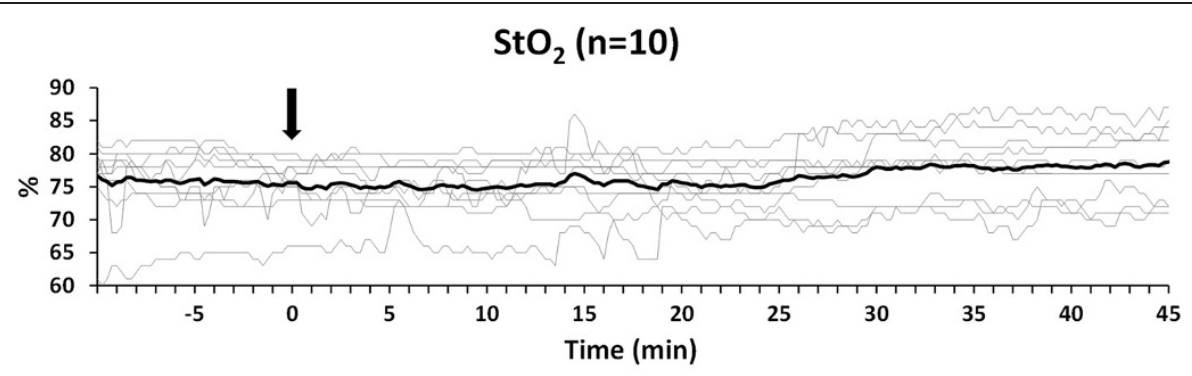

$\Delta \mathrm{Cl}(\mathrm{n}=10)$

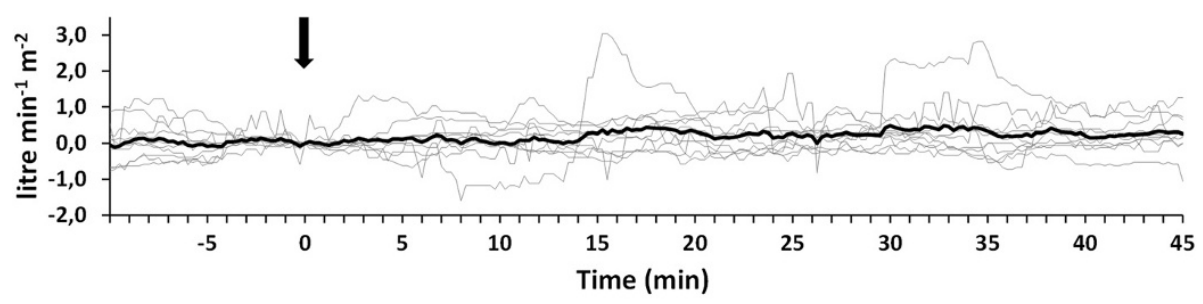

$\Delta \mathrm{ScvO}_{2}(\mathrm{n}=8)$

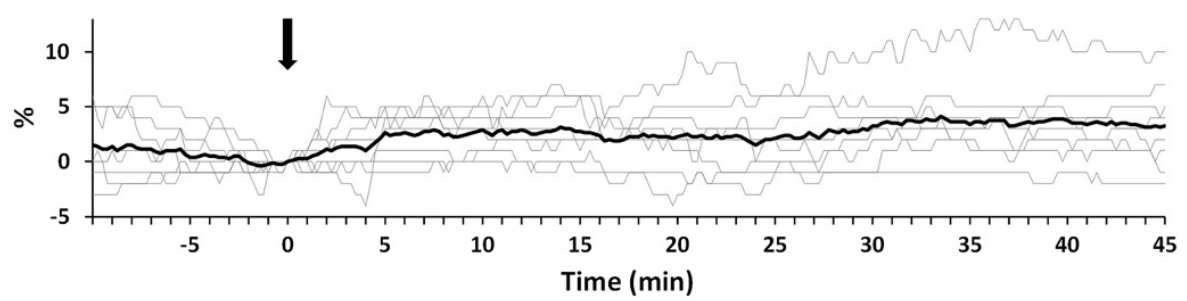

Figure 3 Evolution of the individual patient values (thin lines) and the average values (thick line) of the tissue oxygenation $\left(\mathrm{StO}_{2}\right)$ and the relative change of both cardiac index $(\mathrm{Cl})$, delta $\mathrm{Cl}$, and central venous oxygen saturation $\left(\mathrm{ScvO}_{2}\right)$, delta $\mathrm{ScvO}_{2}$ (data of two patients missing/inadequate due to technical problems). All graphs are synchronized the moment of the first dobutamine administration $($ Time $=0$; arrow). Values are shown from 10 minutes before dobutamine administration until 45 minutes thereafter.

difference between groups in either the amount of RBC transfused $(\mathrm{p}=0.06$ and $\mathrm{p}=0.32$, respectively), in number of patients who received $\mathrm{RBC}$ transfusions $(\mathrm{p}=0.08$ and $\mathrm{p}=0.25$, respectively) or in post transfusion hemoglobine values $(\mathrm{p}=0.73$ and $\mathrm{p}=0.74$, respectively); Table 1 .

\section{Outcome data}

Neither the total amount of complications nor the distribution of complications showed significant differences between groups (Table 2). Eleven patients in the intervention group had no complication, versus 7 in the control group $(p=0.20)$. Also, the median length of stay in various wards and hospital stay were equally distributed. One patient randomized to the intervention group died within 28 days postoperatively due to bowel ischemia noted during relaparotomy. One relaparotomy in the control group revealed leakage after pancreatic surgery.

Based on the average number of postoperative complications between the control and intervention group, a sample size was calculated revealing at least 118 patients
(59 per group) will be needed to gain significant results in a follow-up study, with a power of 0.80 .

\section{Feasibility}

The overall protocol adherence was $96 \%$ in the control group and $92 \%$ in the intervention group (Figure 2). Protocol adherence was determined by assessing the proportion of patients that had to be excluded due to protocol violation and deviation and consequently assessing the number of cases where the protocol was successfully followed. The total exclusion amounted to $20 \%$ (17\% from the control group and $23 \%$ from the intervention group) but exclusion due to protocol deviation or violation was only $6 \%$.

\section{Discussion}

During (high-risk) surgery or during treatment of critically ill patients, the ultimate goal is to maintain or to restore sufficient $\mathrm{StO}_{2}$ since it is a prerequisite of aerobic metabolism. In recent years the clinical use of near 
Table 2 Postoperative outcome

\begin{tabular}{|c|c|c|c|c|c|}
\hline & Control $(n=20)$ & Intervention $(n=20)$ & P-value* & $\operatorname{Dobu}^{+}(n=10)$ & P-value \\
\hline Number of complications (n) & 20 & 11 & 0.23 & 7 & 0.49 \\
\hline Patients without complications (n) & 7 & 11 & 0.34 & 4 & 1.00 \\
\hline Complications & & & 0.35 & & 0.56 \\
\hline Pneumonia & 4 & 5 & & 3 & \\
\hline Respiratory failure & 1 & & & & \\
\hline Cardiac failure & & 1 & & 1 & \\
\hline Supraventricular arrhythmia & 3 & 1 & & & \\
\hline Relaparotomy & 1 & 1 & & 1 & \\
\hline lleus & 3 & 1 & & 1 & \\
\hline Urinary tract infection & 2 & 1 & & 1 & \\
\hline Renal failure & 1 & & & & \\
\hline $\mathrm{TIA}$ & 1 & & & & \\
\hline Delirium & 4 & & & & \\
\hline Hematoma & 1 & & & & \\
\hline Death & & 1 & & & \\
\hline SOFA score & $5[2-12]$ & $6[5-11]$ & 0.73 & $5[5-9]$ & 0.78 \\
\hline TISS score & 25 [21-33] & 26 [22-30] & 0.95 & $28[26-48]$ & 0.96 \\
\hline LOS ICU (hours) & $22[21-45](n=15)$ & $22[17-38](n=15)$ & 0.53 & $22[17-43](n=8)$ & 0.50 \\
\hline LOS PACU (hours) & $18[17-22](n=5)$ & $18[13-22](n=5)$ & 0.75 & $17[17,18](n=2)$ & 0.42 \\
\hline LOS FFD (hours) & 18 [8-37] & 17 [6-23] & 0.39 & 18 [13-36] & 0.70 \\
\hline LOS Hosp (days) & 12 [9-17] & 13 [7-17] & 0.96 & 15 [8-25] & 0.72 \\
\hline
\end{tabular}

$\mathrm{Dobu}^{+}$, subgroup of intervention subjects that did receive dobutamine; SOFA, sequential organ failure assessment; TIA, transient ischemic attack; LOS, length of stay; ICU, intensive care unit; PACU, post anesthesia care unit; FFD, fit for discharge; Hosp, hospital; *Control versus Intervention; ${ }^{\S}$ Control versus Dobu ${ }^{+}$.

infrared spectroscopy (NIRS) to detect tissue oxygenation has increased, especially in the monitoring of critically ill patients and during cardiac surgery [8-10]. Data on the intraoperative use of $\mathrm{StO}_{2}$ in non-cardiac surgery however are scarce. Of note, the study designs differ substantially, which hampers proper comparison of the results. A randomised controlled trial excecuted in a critical care unit showed that postoperative application of stroke volume guided therapy and low-dose inotropic therapy (dopexamine) was associated with improved tissue oxygenation [11]. However, due to study design and insufficient power the investigators were unable to demonstrate an intervention-related reduction in complications rates. Other recent observations revealed that minimum $\mathrm{StO}_{2}$ values in the perioperative setting are inversely associated with outcome [4]. These findings suggest that perioperative optimisation of $\mathrm{StO}_{2}$ values may result in better outcome. The present results cannot fully confirm the abovementioned studies. Despite the category of patients randomised here (all ASA III, age $>65 \mathrm{yr}$ ) who are at elevated risk for lower $\mathrm{StO}_{2}$ values [12] and perioperative morbidity [13] a significant reduction in the number of complications in the intervention group could not be demonstrated in this pilot study. The relatively small number of patients is probably the main reason for this. Another important factor is the fact that only half of the patients randomized for the intervention group actually required dobutamine. Also, in only $40 \%$ of the patients who received dobutamine the predefined $\mathrm{StO}_{2}$ threshold of $80 \%$ was reached permanently. One could argue if dobutamine administration was the most favorable intervention. The increase in myocardial contractility ( $\beta_{1}$ adrenoceptors) and peripheral vasodilation ( $\beta_{2}$ adrenoceptors) generated a modest increase in $\mathrm{StO}_{2}, \mathrm{CI}$ and $\mathrm{ScvO}_{2}$.

Our observations raise another relevant issue, namely the adequacy of the predefined $\mathrm{StO}_{2}$ threshold of $80 \%$ used in this study. $\mathrm{StO}_{2}$ values below $80 \%$ are considered inadequate $[10,14]$ and together with recommendations of the manufacturer a threshold of $80 \%$ seemed reasonable. However, in patients scheduled for non-cardiac surgery, the time averaged $\mathrm{StO}_{2}$ values of approximately $85 \%$ have been described $[4,12]$. These $\mathrm{StO}_{2}$ values are comparable to values seen in healthy volunteers breathing roomair $[14,15]$. Hence, the $\mathrm{StO}_{2}$ threshold used in the present study may have been insufficient and the use 
of a higher $\mathrm{StO}_{2}$ threshold may have led to fewer complications in the intervention group. Abovementioned studies did not describe an optimal $\mathrm{StO}_{2}$ target and therefore another $\mathrm{StO}_{2}$ threshold than $80 \%$, i.e. $85 \%$, may be considered for future studies. Of note, flipside to that coin may be increased use of fluids and vasoactive medication.

We observed a trend of an increased number of RBC transfusion and of more patients in whom RBC transfusion were conducted in the control group compared to the intervention group. Although RBC transfusions were not controlled by study protocol other than a transfusion threshold of $\mathrm{Hb}=5.0 \mathrm{mmol} / \mathrm{L}$ we doubt if the transfusions conducted were of influence on our results. Surely, the effect of RBC transfusions on tissue oxygenation by increasing oxygen delivery is yet unclear $[16,17]$. RBC transfusions elevate the hemoglobin content in the microcirculation but not necessarily the microcirculatory perfusion in cardiac surgery patients [18]. However, considerable interindividual variability exists and the effects of $\mathrm{RBC}$ transfusion on $\mathrm{StO}_{2}$ values seem unpredictable $[19,20]$.

A yet unraveled issue with application of NIRS is the choice of measurement site [21]. For the measurement of peripheral tissue oxygenation multiple measurement sites are available. However, only few sites including the thenar eminence are established [10]. The thenar eminence has a relatively thin fat layer with only minor inter-individual variation. Also, the thenar eminence participates in systemic edema formation to a lesser extent than other skin regions [22] Abovementioned arguments together with the practical perspective from an anesthesists viewpoint explain our choice for the thenar eminence as measurement site. Nevertheless, in general we suspect that $\mathrm{StO}_{2}$ measurements mostly convey information on microcirculatory integrity. It is possible that only after a persisting and sufficiently large change in systemic oxygen balance is reflected in thenar $\mathrm{StO}_{2}$. The main research question is whether the goal-directed $\mathrm{StO}_{2}$ based treatment is correct and well dosed. Based on the present study we are unable to answer that question.

The development of a feasible protocol to determine any significant differences between the study groups remains important. For a study as such where small differences between study groups are observed, it is essential to accurately collect data. The study protocol should be clearly outlined for investigators, as to avoid protocol deviations. We believe that the present study fulfills these criteria. Data were collected continuously, the attending anesthesists were informed comprehensibly and the study protocol was present in the operating room at all times. Studies have shown that protocol adherence becomes one of the main barriers when implementing changes in a clinical setting, with regards to goal directed therapy (GDT) [23-25]. Reasons for low adherence to
GDT include time consumption of conducting the protocol, the tendency of monitoring to be invasive and personal disbelief that such a protocol will result in better outcome [25]. Protocol adherence in this study was $94 \%$ which is considered acceptable $(\geq 75 \%)$ [2]. This can be attributed to the convenient and non-invasive nature and easy interpretation of the obtained data provided by the NIRS equipment [10].

This study has several limitations. First, the observations of the present randomized controlled pilot study are limited to the intraoperative period only. One could argue that the direct postoperative hours are as interesting in efforts to improve outcome after high-risk surgery. Indeed, clinically important fluctuations in the balance between $\mathrm{DO}_{2}$ and $\mathrm{VO}_{2}$ do also occur in the first postoperative hours [26,27] and corresponding hemodynamic interventions seem appropriate. On top of that, postoperative hemodynamic optimisation improves tissue oxygenation [11] and we believe that goal-directed protocols ideally should also cover the early postoperative period. However, because of technical challenges in reliably transporting all the equipment, including the data logging devices, along with the patient to the ICU or PACU, measurements were restricted to the intraoperative period. While our results tend to indicate an advantage of $\mathrm{StO}_{2}$ based goal-directed therapy, the limited power of this pilot restricts any clear recommendations before further larger studies are conducted.

\section{Conclusions}

In conclusion, this is the first randomized controlled study that used $\mathrm{StO}_{2}$ values as a target for hemodynamic optimization in high-risk abdominal surgery patients. Our pilot results are inconclusive on the perioperative optimisation of $\mathrm{StO}_{2}$ values in relation to outcome after high-risk abdominal surgery. Nevertheless, the perioperative strategy described deserves emulation and further research is obligatory to define both the optimal $\mathrm{StO}_{2}$ threshold and intervention to treat or prevent tissue hypoperfusion.

\section{Abbreviations \\ BIS: Bispectral monitoring; $C_{e}$ t: Effect site concentration; Cl: Cardiac index; CO: Cardiac output; CVP: Central venous pressure; $\mathrm{DO}_{2}$ : Oxygen delivery; FFD: Fit for discharge; ICU: Intensive care unit; NIRS: Near infrared spectroscopy; PACU: Post-anesthesia care unit; RBC: Red blood cell; $\mathrm{rSO}_{2}$ : Regional cerebral oxygenation; $\mathrm{SCvO}_{2}$ : Central venous oxygen saturation; $\mathrm{SpO}_{2}$ : Oxygen saturation $\mathrm{StO}_{2}$ : Tissue oxygenation; $\mathrm{TCl}$ : Target-controlled infusion.}

Competing interests

The authors declare that they have no competing interests.

\section{Authors' contributions}

PB drafted the manuscript, participated in its design and coordination, and performed statistical analysis. JV responsible for data logging system and acquisition of continuous patient data and helped to draft the manuscript. MP responsible for processing continuous data, performed statistical analysis and helped to draft the manuscript. AK participated in the design of the 
study and helped to draft the manuscript. TS conceived of the study and participated in its design and coordination and helped to draft the manuscript. All authors read and approved the final manuscript.

\section{Acknowledgements}

The authors would like to thank research nurse Ans Hagenaars for her invaluable help in the acquisition of patient data.

This work was supported by an unrestrictive grant from Hutchinson Technology, Hutchinson, MN, USA.

Received: 27 July 2014 Accepted: 8 December 2014

Published: 16 December 2014

\section{References}

1. Pearse RM, Harrison DA, James P, Watson D, Hinds C, Rhodes A, Grounds RM, Bennet ED: Identification and characterization of the high-risk surgical population in the United Kingdom. Crit Care 2006, 10:R81.

2. Scheeren TW, Wiesenack C, Gerlach H, Marx G: Goal-directed intraoperative fluid therapy guided by stroke volume and its variation in high-risk surgical patients: a prospective multicentre study. J Clin Monit Comput 2013, 27:225-233.

3. Giglio MT, Marucci M, Brienza TM: Goal-directed haemodynamic therapy and gastrointestinal complications in major surgery: a meta-analysis of randomized controlled trials. Br J Anaesth 2009, 103:637-646.

4. Abdelmalak BB, Cata JP, Bonilla A, You J, Kopyeva T, Vogel D, Campbell S, Sessler DI: Intraoperative tissue oxygenation and postoperative outcomes after major non-cardiac surgery: an observational study. $\mathrm{Br} J$ Anaesth 2013, 110:241-249.

5. Ehrenfeld JM, Funk LM, Van Schalkwyk J, Merry AF, Sandberg WS, Gawande A: The incidence of hypoxemia during surgery: evidence from two institutions. Can J Anaesth 2010, 57:888-897.

6. Cannesson M, Pestel G, Ricks C, Hoeft A, Perel A: Hemodynamic monitoring and management in patients undergoing high risk surgery: a survey among North American and European anesthesiologists. Crit Care 2011, 15:R197.

7. Faul F, Erdfelder E, Lang AG, Buchner A: G Power 3: a flexible statistical power analysi program for the social, behavioral, and biomedical sciences. Behav Res Med 2007, 39:175-191.

8. Schwarte LA, Stevens MF, Ince C: Hepatosplanchnic failure: splanchnic perfusion and oxygenation in critical illness. In Yearbook of intensive care and emergency medicine. Edited by Vincent JL. Berlin: Springer; 2006:627-640

9. Brun-Buisson C, Doyon F, Carlet J, Dellamonica P, Gouin F, Lepoutre A, Mercier JC, Offenstadt G, Régnier B: Incidence, risk factors, and outcome of severe sepsis and septic shock in adults. A multicenter prospective study in intensive care units. French ICU Group for Severe Sepsis. JAMA 1995, 274:968-974

10. Scheeren TWL, Schober P, Schwarte LA: Monitoring tissue oxygenation by near infrared spectroscopy (NIRS): background and current applications. J Clin Monit Comput 2012, 26:279-287.

11. Jhanji S, Vivian-Smith A, Lucena-Amaro S, Watson D, Hinds CJ, Pearse RM: Haemodynamic optimisation improves tissue microvascular flow and oxygenation after major surgery: a randomized controlled trial. Crit Care 2010, 14:R151

12. Spruit RJ, Schwarte LA, Hakenberg O, Scheeren TWL: Association of intraoperative tissue oxygenation with suspected risk factors for tissue hypoxia. J Clin Monit Comput 2013, 27:541-550.

13. Boersma E, Kertai MD, Schouten O, Bax JJ, Noordzij P, Steyerberg EW, Schinkel AF, van Santen M, Simoons ML, Thomson IR, Klein J, van Urk H, Poldermans D: Perioperative cardiovascular mortality in noncardiac surgery: validation of the Lee risk index. Am J Med 2005, 118:1134-1141.

14. Crookes BA, Cohn SM, Bloch S, Amortegui J, Manning R, Li P, Proctor MS, Hallal A, Blackbourne LH, Benjamin R, Soffer D, Habib F, Schulman Cl, Duncan R, Proctor KG: Can near-infrared spectroscopy identify the severity of shock in trauma patients? J Trauma 2005, 58:806-813.

15. Larsson A, Uusijarvi J, Eksborg S, Lindhol P: Tissue oxygenation measured with near-infrared spectroscopy during normobaric and hyperbaric oxygen breathing in healthy subjects. Eur J Appl Physiol 2010, 109:757-761.

16. Dietrich KA, Conrad SA, Hebert CA, Levy GL, Romero MD: Cardiovascular and metabolic response to red blood cell transfusion in critically ill volume-resuscitated nonsurgical patients. Crit Care Med 1990, 18:940-944.
17. Lorente JA, Landin L, Pablo R, Renes E, Rodriquez-Diar R, Liste D: Effects of blood transfusion on oxygen transport variables in sepsis. Crit Care Med 1993, 21:1312-1318.

18. Yuruk K, Almac E, Bezemer R, Goedhart P, De Mol B, Ince C: Blood transfusions recruit the microcirculation during cardiac surgery. Transfusion 2011, 51:961-967.

19. Creteur J, Neves AP, Vincent JL: Near-infrared spectroscopy technique to evaluate the effects of red blood cell transfusion on tissue oxygenation. Crit Care 2009, 13:S11.

20. Yuruk K, Bartels SA, Milstein DM, Bezemer R, Biemond BJ, Ince C: Red blood cell transfusions and tissue oxygenation in anemic hematology outpatients. Transfusion 2012, 52:641-646.

21. van Beest PA, Scheeren TWL: Tissue oxygen saturation as a goal, but when and where should we measure it? J Clin Monit Comput 2013, 27:211-213.

22. Poeze M: Tissue oxygenation assessment using near-infrared spectroscopy during severe sepsis: confounding effects of tissue edema on $\mathrm{StO}_{2}$ values. Intensive Care Med 2006, 32:788-789.

23. Crowe CA, Mistry CD, Rzechula K, Kulstad CE: Evaluation of a modified early goal-directed therapy protocol. Am J Emerg Med 2010, 28:689-693.

24. Mikkelsen ME, Gaieski DF, Goyal M, Miltiades AN, Munson JC, Pines JM, Fuchs BD, Shah CV, Bellamy SL, Christie JD: Factors associated with nonadherence to early goal-directed therapy in the ED. Chest 2010, 138:551-558.

25. O'Neill R, Morales J, Jule M: Early goal-directed therapy (EGDT) for severe sepsis/septic shock: which components of treatment are more difficult to implement in a community-based emergency department? J Emerg Med 2012, 42:503-510.

26. Pearse R, Dawson D, Fawcett J, Rhodes A, Grounds RM, Bennett ED: Changes in central venous saturation after major surgery, and association with outcome. Crit Care 2005, 9:R694.

27. Collaborative Study Group on Perioperative ScvO2 Monitoring: Multicentre study on peri- and postoperative central venous oxygen saturation in high-risk surgical patients. Crit Care 2006, 10:R158.

doi:10.1186/1471-2253-14-122

Cite this article as: van Beest et al: Tissue oxygenation as a target for goal-directed therapy in high-risk surgery: a pilot study. BMC Anesthesiology 2014 14:122.

\section{Submit your next manuscript to BioMed Central and take full advantage of:}

- Convenient online submission

- Thorough peer review

- No space constraints or color figure charges

- Immediate publication on acceptance

- Inclusion in PubMed, CAS, Scopus and Google Scholar

- Research which is freely available for redistribution 